\title{
Relación entre variables socioeconómicas y tolerancia al riesgo financiero de inversionistas de Sonora, México
}

\author{
González-Vejar Roberto Luis ${ }^{1 *}$, León-Balderrama Jorge Inés ${ }^{2}$, Garibay-Escobar Sergio ${ }^{3}$ \\ 1. Maestro en Administración Pública y candidato a doctor en Desarrollo Regional por el Centro de Investigación en Alimentación y \\ Desarrollo, A.C. (CIAD). \\ 2. Centro de Investigación en Alimentación y Desarrollo A.C. (CIAD). Coordinación de Desarrollo Regional \\ 3. Universidad de Sonora. Doctor en Administración Pública
}

\section{Resumen}

El presente estudio es un diseño no experimental transversal comparativo que tuvo como objetivo asociar y comparar variables socioeconómicas y la tolerancia al riesgo financiero de inversionistas de las ciudades de Guaymas y Hermosillo, Sonora, México. A partir de un muestreo bola de nieve se seleccionaron a 147 inversionistas con patrimonio por encima de 7 millones de dólares. Por medio de consentimiento informado, se les aplicó un cuestionario de tolerancia al riesgo, horizonte de tiempo y objetivos financieros realizado a través de investigaciones sobre el tema de riesgos en las inversiones. Los resultados indicaron que la muestra seleccionada presenta niveles de bajos a moderados de tolerancia al riesgo. De igual manera se encontró que variables socio económicas, como el nivel de escolaridad, edad y patrimonio se relacionan con la tolerancia al riesgo. Los hallazgos del estudio son de particular relevancia para los asesores-planificadores financieros, las organizaciones profesionales y los reguladores de la industria.

Palabras clave: Tolerancia al riesgo, portafolio de inversiones, percepción de riesgo.

\section{Relationship between socio-economic variables and financial risk tolerance of investors in Sonora, Mexico}

\begin{abstract}
The present study is a non-experimental comparative cross-sectional design that aimed to associate and compare socioeconomic variables and tolerance to financial risk of investors from the cities of Guaymas and Hermosillo, Sonora, Mexico. From a snowball sampling, 147 investors were selected with wealth above 7 million dollars. By means of informed consent, a risk tolerance questionnaire, time horizon and financial objectives were applied through research on the issue of investment risks. The results indicated that the selected sample presents low to moderate levels of risk tolerance. Similarly, it was found that social economic variables such as level of schooling, age and wealth are related to risk tolerance. It was concluded that the study's findings are of particular relevance to financial planners-advisers, professional organizations (of financial services) and industry regulators
\end{abstract}

Keywords: Risk tolerance, investment portfolio, risk perception.

*Autor para envío de correspondencia: Centro de Investigación en Alimentación y Desarrollo, A.C. (CIAD). Real del Arco 208, colonia Villa Satélite, Hermosillo, Sonora. C.P. 83200 E-mail: rlgv11@hotmail.com

(c) 2019 Editorial UNISON — URN. Derechos reservados. 


\section{INTRODUCCIÓN}

La palabra italiana "risicare", que significa atreverse, el riesgo es más una opción que un destino (Bernstein, 1996, pág. 2). El riesgo tiene que ver con las acciones que las personas se atreven a tomar, que dependen en gran medida de la libertad para tomar tales decisiones. Koh y Fong (2011, pág. 22) refieren hasta cuatro tipos de riesgos en los aspectos éticos, sociales, físicos y, por último, los financieros, este último resulta el foco de presente estudio. La tolerancia para los riesgos financieros y no financieros "se refiere a la medida en que las personas son psicológicamente receptivas a diversas decisiones inciertas que afectan su bienestar social, ético, físico o sea financiero" (Koh \& Fong, 2011, pág. 23). Se puede decir que la tolerancia al riesgo "es la suma de todas las compensaciones de miedo/avaricia disponibles" (Finametrica, 2015, pág. 1). Finamétrica (2015) indica que lo anterior incluye compensaciones entre aprovechar al máximo las oportunidades y asegurar el bienestar financiero, las compensaciones entre el arrepentimiento de evitar las pérdidas en las que se incurre por tomar demasiado riesgo y las ganancias anormales que se pierden al no tomar lo suficiente. Por lo tanto, la tolerancia al riesgo se define mejor como "la medida en que una persona elige arriesgarse a experimentar un resultado menos favorable en la búsqueda de un resultado más favorable" (Hallahan, Faff y Mckenzie, 2004, pág. 58).

Este estudio proporciona una revisión conceptual de la Tolerancia al Riesgo Financiero (TRF), sus componentes, determinaciones, cómo puede medirse y el vínculo con diversas variables socioeconómicas. Esto ayuda a crear una base para comprender qué es la tolerancia al riesgo y reconocer que puede aplicarse a diversos tipos, ya sean financieros o no financieros.

Las operaciones en los negocios y el entorno del diario vivir, están rodeados de incertidumbre y la búsqueda de grandes utilidades conlleva riesgo. Según Urteaga \& Izagirre (2013, pág. 148) "el riesgo es la consecuencia aleatoria de una situación, pero bajo la perspectiva de una amenaza o de un posible perjuicio", por otro lado, el riesgo se puede definir como "El efecto de la incertidumbre sobre los objetivos" (ICONTEC, 2009, pág. 4).

Nguyena, Gallery y Newton (2017) indican que, en la actualidad, un alto número de inversionistas poco sofisticados necesitan tomar decisiones sensatas. Los mismos autores refieren que como la mayoría de las inversiones están asociadas con algunos niveles de riesgo, es importante que éstas sean compatibles con los perfiles de riesgo que presentan. Debido a que tales perfiles a menudo son complejos y requieren mucho tiempo para analizarse, así como herramientas analíticas y cuantitativas, un número cada vez mayor de inversores recurren a asesores financieros para obtener sus servicios.
En este sentido, un concepto comúnmente utilizado en las investigaciones sobre riesgo es el de "tolerancia al riesgo", Grable (2000, pág. 625) lo define como "la cantidad máxima de incertidumbre que alguien está dispuesto a aceptar al tomar una decisión financiera". Esta definición ha sido ampliamente utilizada en finanzas personales y de consumo (Grable, 2008).

El fenómeno de la tolerancia al riesgo supone por definición un cierto nivel de aceptación o rechazo al riesgo por parte de una persona que invierte en los mercados financieros $\mathrm{o}$, en un negocio o proyecto. A la hora de calificar a un individuo como opuesto al riesgo en el mundo de las decisiones de inversión es necesario valorar sus preferencias como un aspecto crucial a tener presente (Meyer y Meyer. 2007).

Stangler (2010) refiere que las personas que toleran el riesgo se concentran dependiendo de la industria y de la región; de igual forma, también indica que existen regiones que están avanzando a pasos agigantados y otras que se están quedando rezagadas debido a que los tomadores de riesgo invierten su capital en diferentes proyectos. Glaeser, Kerr y Ponzeto (2010) aluden que las ciudades con altos niveles de emprendedores y de inversionistas tienen mayor crecimiento de empleo que aquellas que dependen más de negocios existentes. Las compañías con alto crecimiento startups tecnológicas - crean un mayor número de empleos que las ya estables.

En relación a lo anterior, Cortés (2016), indica que al momento de realizar una decisión financiera, el nivel de riesgo tolerado por los inversionistas debe de tener en consideración no sólo cuestiones subjetivas (actitud frente al riesgo) que obedecen a razones psicológicas, sino también cuestiones objetivas, como la capacidad que tienen los inversionistas de asumir el riesgo elegido y el ciclo de vida en el que se encuentran.

El riesgo que un inversionista puede permitirse asumir depende entre otros factores de su situación financiera total, las responsabilidades específicas de cada inversionista, edad, educación y personalidad (Kannadhasan, Aramvalarthan y Goyal, 2016).

Por lo anterior, se parte de las preguntas: ¿Qué características socioeconómicas tienen los inversionistas de dos Ciudades de Sonora, México; Guaymas y Hermosillo? ¿Qué nivel de tolerancia al riesgo presentan los inversionistas? ¿Cuáles factores socioeconómicos se relacionan con la tolerancia al riesgo financiero de inversionistas de Guaymas y Hermosillo, Sonora, México? 


\section{MARCO CONCEPTUAL}

El término tolerancia al riesgo se define y utiliza de diferentes maneras. Si la tolerancia al riesgo es una característica estable de un inversionista determinado o también considera las circunstancias externas (por ejemplo, las crisis económicas o el dominio de la decisión) depende de cómo se define y mide. Este resumen se centra en una definición que prevalece en la comunidad de profesionales, es decir, la disposición de un inversor a asumir el riesgo percibido (Davies 2017) o la compensación que un inversor está dispuesto a hacer entre el riesgo percibido y el retorno esperado de diferentes opciones de inversión (Grable 2017). Esta definición deriva de una interpretación psicológica del marco de riesgo - retorno de la teoría clásica de la cartera (Markowitz, 1952). Weber y Milliman (1997) tratan al concepto como una actitud hacia el riesgo y deslinda esta variable actitudinal pura de las percepciones de riesgos y rendimientos: variables psicológicas propias y distintas del valor esperado y la varianza de la distribución de los posibles resultados.

La tolerancia al riesgo financiero es un concepto utilizado comúnmente entre los asesores financieros. Cuando se usa en términos generales, se le alude como un conjunto para varios conceptos relacionados con el riesgo (Weber y Klement, 2018). Es importante señalar, sin embargo, que la tolerancia al riesgo financiero tiene un significado muy específico. Cordell (2001) indicó que el concepto es el grado máximo de incertidumbre que alguien está dispuesto a aceptar cuando toma una decisión financiera que conlleva la posibilidad de una pérdida. Esta declaración coincide con la definición de la Organización Internacional de Normalización (2006) de que la tolerancia al riesgo financiero es el grado en que alguien está dispuesto a experimentar un resultado menos favorable en la búsqueda de un resultado con atributos más favorables.

La tolerancia al riesgo financiero es distinta de conceptos tales como preferencia de riesgo, percepción del riesgo, capacidad de riesgo, necesidad de riesgo o compostura de riesgo. Cada uno de estos conceptos es un aporte esencial para el desarrollo del perfil de riesgo de una persona; sin embargo, estos términos no son intercambiables.

A continuación, se presentan una serie de definiciones siguen la nomenclatura elaborada por Nobre y Grable (2015), quienes seleccionaron la literatura para los marcos de definición de conceptos relacionados con el riesgo:

- Aversión al riesgo, lo contrario de tolerancia al riesgo.

- Capacidad de riesgo, una evaluación objetiva de la capacidad financiera de un individuo para soportar una pérdida financiera.
- Compostura al riesgo, la propensión de un individuo a comportarse de manera consistente.

- Necesidad de riesgo, La cantidad de riesgo que un individuo debe tomar para alcanzar un objetivo financiero; Normalmente se basa en una tasa de retorno requerida predeterminada.

- Percepción de riesgo, Una evaluación subjetiva, basada en una valoración cognitiva, del riesgo de un resultado de una decisión a realizar.

- Preferencia al riesgo, la sensación general de un individuo de que una situación es mejor que otra.

- Perfil de riesgo, una mezcla de factores que ayudan a moldear el comportamiento de riesgo de un individuo.

- Tolerancia al riesgo, grado máximo de incertidumbre que alguien está dispuesto a aceptar cuando toma una decisión financiera que conlleva la posibilidad de una pérdida.

En el contexto de la gestión de portafolios, una forma habitual de constituir la tolerancia al riesgo como una entrada única en el perfil de riesgo general del cliente. Klement (2018) argumentó que la tolerancia al riesgo es solo uno de los varios factores que conforman el perfil de riesgo de un cliente. Otros factores incluyen la edad, los objetivos de inversión, el horizonte de tiempo, la experiencia y la capacidad de riesgo. La combinación de estos factores, según la evaluación de un asesor financiero, determina la combinación adecuada de asignación de activos. Aunque el objetivo del presente trabajo no es realizar perfiles de riesgo, si se retomaran los objetivos de inversión y horizonte del tiempo en el análisis de la tolerancia al riesgo financiero.

Una vez diferenciado el concepto de tolerancia al riesgo, el presente trabajo parte del objetivo general de asociar y comparar variables socioeconómicas con los niveles de tolerancia al riesgo financiero de inversionistas de las ciudades de Guaymas y Hermosillo, Sonora, México.

De igual manera, se busca alcanzar los siguientes objetivos específicos:

- Caracterizar a los inversionistas de dos ciudades de Sonora, México; Guaymas y Hermosillo.

- Describir la tolerancia al riesgo de los inversionistas.

- Asociar variables socioeconómicas con la tolerancia al riesgo.

- Comparar la tolerancia al riesgo a partir de la creación de conglomerados de riesgo con variables socioeconómicas. 


\section{METODOLOGÍA}

\section{Tipo de estudio}

Se presenta un diseño no experimental de tipo transversalcomparativo.

\section{Participantes}

A partir de un muestreo bola de nieve, participaron 147 empresarios del Estado de Sonora, el 78.9\% $(n=116)$ reside en la Ciudad de Hermosillo y el resto en Guaymas, con base en la clasificación del Research Institute del banco Credit Suisse, en su estudio "Global Wealth Databook 2017" (Noviembre 2017) y de acuerdo a encuestas y cuestionarios realizados por los grandes bancos mundiales, donde una persona se "siente rica" a partir de 4 a 7 millones de dólares, en base a eso, se seleccionaron a empresarios inversionistas que posean un capital de al menos 4 millones de dólares americanos.

\section{Instrumentos}

El instrumento a utilizar, en este proyecto de investigación, es un cuestionario diseñado para evaluar la tolerancia al riesgo de un inversionista y para obtener un conocimiento profundo del objetivo de su inversión, permitiéndonos conocer el tipo de inversiones a los que el inversionista es susceptible. El objetivo de inversión se basa en diversos factores, como el horizonte de tiempo, metas financieras y tolerancia al riesgo. Se retomó el cuestionario realizado por Nguyen et al. (2017) para evaluar la tolerancia al riesgo además, se incorporaron reactivos del cuestionario de perfil de inversionista elaborado por LPL Financial para valorar el horizonte de tiempo y el objetivo de la inversión.

Horizonte de tiempo. El horizonte de tiempo de la inversión para logar las metas financieras deseadas. Contiene 3 reactivos como ¿̇uál es tu principal meta financiera?

Tolerancia al riesgo. Presenta 7 reactivos tales como "Acabas de recibir una gran cantidad de dinero ¿Cómo la invertirías? (1) Invertiría en algo que me ofrezca actualmente, un moderado ingreso y que fuera muy conservador (2) Invertiría en algo que me ofrezca actualmente, un alto ingreso con moderada cantidad de riesgo (3) Invertiría en algo que me ofrezca, un alto retorno (4) no sustancial apreciación de capital aunque tenga una alta cantidad de riesgo". Puntajes de 1 a 7 indican muy poca tolerancia al riesgo, de 8 a 14 baja tolerancia, 15 a 18 moderada tolerancia 19 a 22 alta tolerancia y de 23 a 27 muy alta tolerancia.

Objetivo de la inversión. El objetivo del inversionista se encuentra directamente relacionado con el tipo de inversión, en caso de que existan cambios en el objetivo, las inversiones deben de evaluarse. Está constituida por 5 reactivos como: ¿De hoy a 5 años cuánto espera que sea el valor de su portafolio de inversión? (1) Me preocupa más el ingreso actual (2) El mismo o un poco mayor al valor actual (3) Mayor al valor actual (4) Substancialmente mayor al valor actual.

El instrumento posee diversas opciones de respuesta por reactivo, principalmente se presenta una escala que va el 1 al 4 y al finalizar se realizan sumatorias para generar un nivel de tolerancia al riesgo, horizonte de tiempo y objetivos de inversión. Respecto a la confiabilidad del instrumento, se sometió a la prueba de alfa de Cronbach, se obtuvieron puntajes mayores a 0.75 en las dimensiones del test.

\section{Procedimiento}

Se seleccionaron a inversionistas de dos ciudades del estado que tuvieran un patrimonio de al menos 4 millones de dólares. Mediante consentimiento informado los empresarios accedieron a participar en el estudio. Los cuestionarios se aplicaron entre el 15 de junio y el 30 de octubre del 2018. El tiempo de aplicación del instrumento fue de aproximadamente 8 minutos. Una vez que se levantó la información, se procedió a realizar una base de datos en el programa SPSS versión 2.0. Se utilizó un análisis de frecuencias, también pruebas paramétricas como la t de Student y r de Pearson; de igual manera, se utilizó la prueba multivariada de $\mathrm{k}$ medias con el fin de crear conglomerados a partir de los puntajes de tolerancia al riesgo.

\section{RESULTADOS}

Las empresas de las que son propietarios los inversionistas que conforman la muestra, tienen un giro principalmente de bienes y servicios en ambas ciudades, les sigue el comercio y las actividades primarias como la agricultura y la pesca. Respecto al tipo de adquisición, la gran mayoría son fundadores de sus empresas. En lo que respecta a la fundación de las mismas, las empresas de Guaymas son más antiguas con 42 años en promedio mientras que las de Hermosillo tienen alrededor de 33 años.

La actividad económica o giro de las empresas que poseen los inversionistas conlleva a que el sector de pesca es el que más contribuye a las fortunas de los empresarios, le sigue construcción y agricultura respectivamente (Ver Tabla 2). 
Tabla 1. Descripción de las empresas y/o actividades de los inversionistas de dos ciudades de Sonora

\begin{tabular}{|c|c|c|}
\hline $\begin{array}{l}\text { Información de la } \\
\text { empresa }\end{array}$ & Hermosillo & Guaymas \\
\hline \multicolumn{3}{|l|}{ Giro } \\
\hline Bienes y servicios & $46 \%(n=54)$ & $25.8 \%(n=8)$ \\
\hline Comercio & $30.2 \%(n=35)$ & $32.3 \%(n=10)$ \\
\hline Agricultura & $16.45 \%(n=19)$ & $16.1 \%(n=5)$ \\
\hline Construcción & $6.9 \%(8)$ & \\
\hline Pesca & & $25.8 \%(n=8)$ \\
\hline \multicolumn{3}{|l|}{ Tipo de adquisición } \\
\hline Fundador & $85.3 \%(n=99)$ & $74.2 \%(n=23)$ \\
\hline Heredó & $14.7 \%(n=17)$ & $19.4 \%(n=6)$ \\
\hline Compró & & $6.5 \%(n=2)$ \\
\hline Fecha de adquisición & $1986.56 \quad$ (DS. & 1977.29 \\
\hline Total & $\begin{array}{c}15.54) \\
116\end{array}$ & $\begin{array}{c}\text { (DS. 23./7) } \\
31\end{array}$ \\
\hline
\end{tabular}

Notas. 1) DS= Desviación estándar; 2) tabla de elaboración propia, con base en los resultados de los cuestionarios aplicados.

Tabla 2. Valor aproximado de las fortunas de los inversionistas por tipo de actividad a la que se dedican.

\begin{tabular}{lcc|}
$\begin{array}{l}\text { Actividades de los } \\
\text { inversionistas }\end{array}$ & $\begin{array}{c}\text { Valor aproximado } \\
\text { de sus fortunas }\end{array}$ & $\begin{array}{c}\text { Número de } \\
\text { inversionistas }\end{array}$ \\
\hline Comercio & $209,856,666$ & 45 \\
\hline Bienes y servicios & $376,877,258$ & 62 \\
\hline Agricultura & $731,972,916$ & 24 \\
\hline Construcción & $1,002,300,000$ & 8 \\
\hline Pesca & $1,027,893,750$ & 8
\end{tabular}

Nota. Tabla de elaboración propia, en base a los resultados de los cuestionarios aplicados.

En cuanto a variables específicas de los inversionistas, el $100 \%$ de ellos son hombres en ambas ciudades, su edad promedio fue entre los 64 y 67 años. El 58\% posee un nivel educativo superior y el $36.1 \%$ preparatoria. Respecto al valor neto de las fortunas que poseen los inversionistas, la de los empresarios de Guaymas es mayor.

Tabla 3. Descripción de los inversionistas de dos ciudades de Sonora

\begin{tabular}{lcccc} 
Variables & \multicolumn{2}{c}{ Hermosillo } & \multicolumn{2}{c}{ Guaymas } \\
& Media & D.S & Media & DS \\
Edad & 64.04 & 13.89 & 66.45 & 12.54 \\
$\begin{array}{l}\text { Valor } \\
\text { aproximado de } \\
\text { su fortuna }\end{array}$ & $\begin{array}{c}387.8 \\
\text { millones }\end{array}$ & $\begin{array}{c}748 \\
\text { millones }\end{array}$ & $\begin{array}{c}699.5 \\
\text { millones }\end{array}$ & $\begin{array}{c}567.2 \\
\text { millones }\end{array}$ \\
\hline
\end{tabular}

Nota. DS = Desviación estándar.

En relación a la tolerancia al riesgo financiero, los inversionistas de las dos ciudades de Sonora se encuentran con puntajes que los ubican en baja tolerancia. Presentan un horizonte de tiempo de corto a medio plazo sobre la inversión. Sus objetivos de inversión no los llevan a enfocarse en el crecimiento, los puntajes indican se centran en la retención del capital e ingreso actual.
Tabla 4. Tolerancia al riesgo, horizonte de tiempo y objetivos de los inversionistas

\begin{tabular}{lcccc} 
Variables & \multicolumn{2}{c}{ Hermosillo } & \multicolumn{2}{c}{ Guaymas } \\
& Media & D.S & Media & DS \\
\hline Horizonte de tiempo & 3.96 & 1.00 & 3.48 & .96 \\
\hline Tolerancia al riesgo & 14.18 & 3.56 & 13.48 & 1.62 \\
\hline Objetivos de inversión & 7.25 & 2.56 & 6.22 & .99
\end{tabular}

Notas. DS = Desviación estándar.

En la tabla 5 se puede observar que la tolerancia al riesgo se correlacionó con el nivel educativo de los inversionistas $(r=.180$, sig. 047) lo que indica que, a mayor escolaridad de los empresarios, mayor tolerancia al riesgo existirá. La edad también resultó con asociación respecto a la tolerancia (r. 186), aspecto que se describirá más adelante. De igual manera, el giro de la empresa se asocia con la medida de riesgo (r.256, sig. 000). Cabe mencionar que, aunque no es objetivo del presente trabajo, el nivel educativo fue una de las variables que mayor se asoció con indicadores relevantes como la fortuna y el giro de la empresa.

Tabla 5. Correlaciones paramétricas entre la tolerancia al riesgo financiero y variables socioeconómicas de los inversionistas

\begin{tabular}{lcccccc} 
& \multicolumn{7}{c}{ Variables } \\
& $\mathbf{1}$ & $\mathbf{2}$ & $\mathbf{3}$ & $\mathbf{4}$ & $\mathbf{5}$ & $\mathbf{6}$ \\
\hline $\begin{array}{l}\text { Tolerancia } \\
\text { al riesgo (1) }\end{array}$ & $\mathbf{1}$ & $.180^{*}$ & -.084 & $.186^{*}$ & .037 & $.256^{* *}$ \\
\hline $\begin{array}{l}\text { Nivel } \\
\text { educativo (2) }\end{array}$ & --- & 1 & $.225^{* *}$ & $.364^{* *}$ & -.040 & $.425^{* *}$ \\
\hline Fortuna (3) & --- & --- & 1 & .009 & -.012 & -.111 \\
\hline Edad (4) & --- & --- & --- & 1 & $.266^{* *}$ & $-.409^{* *}$ \\
\hline $\begin{array}{l}\text { Tipo de } \\
\text { adquisición (5) }\end{array}$ & --- & --- & --- & --- & 1 & -.020 \\
\hline $\begin{array}{l}\text { Giro de la } \\
\text { empresa (6) }\end{array}$ & --- & --- & --- & --- & --- & 1 \\
\hline
\end{tabular}

$\mathrm{p}^{*}=.05 ; \mathrm{p}^{* *}=.000$

Nota. Tabla de elaboración propia, en base a los resultados de los cuestionarios aplicados.

A través de la prueba de $\mathrm{k}$ medias se realizó un análisis de clúster tomando como referencia los puntajes de tolerancia al riesgo financiero, con 9 iteraciones se crearon dos conglomerados; al primero se le llamo inversionistas con baja tolerancia al riesgo y al segundo inversionistas con moderada tolerancia al riesgo. Cabe mencionar que debido a aparente homogeneidad de la muestra no se hizo distinción entre la ciudad de residencia, se tomaron únicamente los valores de tolerancia para realizar la prueba multivariada.

En la tabla 6 se muestra que el nivel educativo marca diferencias significativas en cuanto a la tolerancia al riesgo se refiere $(t=2.43$; sig. 017$)$ aunque la diferencia 
entre la escolaridad es baja, los resultados indican que los inversionistas con mayor nivel educativo tienen mayor tolerancia. También se obtuvo que a menor edad mayor tolerancia al riesgo.

Tabla 6. Comparaciones paramétricas por conglomerado y variables socioeconómicas.

\begin{tabular}{|c|c|c|c|c|c|}
\hline \multirow[t]{2}{*}{ Variables } & \multicolumn{2}{|c|}{ Baja tolerancia } & \multicolumn{3}{|c|}{ Moderada tolerancia } \\
\hline & Media & D.S. & Media & D.S. & Prueba $t$ \\
\hline $\begin{array}{l}\text { Tolerancia al } \\
\text { riesgo }\end{array}$ & 12.65 & 1.97 & 18.16 & 2.81 & $13.07^{* *}$ \\
\hline $\begin{array}{l}\text { Nivel } \\
\text { educativo }\end{array}$ & 3.37 & .68 & 3.67 & .60 & $2.43^{*}$ \\
\hline Fortuna & 461 millones & & 428 millones & & .151 \\
\hline Edad & 68.45 & 13.44 & 63.22 & 13.55 & $2.04^{*}$ \\
\hline Total & $\mathrm{n}=\mathbf{1 1 0}$ & & $\mathrm{n}=37$ & & \\
\hline
\end{tabular}

elaboración propia, en base a los resultados de los cuestionarios aplicados.

\section{DISCUSIÓN Y CONCLUSIONES}

Se partió de diversos objetivos que tuvieron como fin asociar y comparar variables socioeconómicas con la tolerancia al riesgo financiera externada por los inversionistas de Guaymas y Hermosillo, Sonora, México. Primero que nada, se puede decir que las características de los inversionistas es que la mayoría de ellos están por entrar a la adultez tardía. El horizonte de tiempo resulta una variable estrictamente relacionada con la tolerancia al riesgo, al ser la mayoría de los inversionistas de edad avanzada, existe la posibilidad de que se busquen retornos de inversión a corto plazo y exista una baja tolerancia al riesgo (Klement, 2018), tal y como fue el resultado alcanzado en la presente investigación.

Las actividades primordiales en cuanto a las fortunas o riquezas creadas por los inversionistas resultaron en el sector primario, tanto la pesca, cultivo y ganadería resultan actividades que actualmente generan riquezas en el Estado tal y como especificó la Secretaría de Agricultura Ganadería Desarrollo Rural Pesca y Alimentación (2015).

Se encontró una relación entre variables socioeconómicas con la tolerancia al riesgo mostrada, los resultados se sostienen por lo encontrado por Mahmood et al. (2011) propusieron un modelo en el cual identificaron algunos factores socioeconómicos, demográficos y de actitud que pueden influir la propensión para buscar o evitar el riesgo y la percepción del mismo. Estos factores incluyeron género, estado civil, experiencias anteriores, escolaridad, diferencias en políticas de regulación, asimetría de información y la búsqueda de sensaciones.

Se concuerda con Nguyen et al., (2017) en relación a no explorar únicamente el concepto de tolerancia al riesgo, también resulta importante reforzarlo con percepción del riesgo; en comparación con la tolerancia al riesgo, la percepción del riesgo puede cambiar fácilmente con el tiempo, ya que refleja cómo las personas perciben un producto de inversión (Roszkowski y Davey, 2010). Los asesores financieros pueden intervenir en este proceso de toma de decisiones al afectar la percepción de sus clientes y ayudar a los clientes a tomar decisiones acertadas.

Un aspecto relevante, es que los inversionistas con una fortuna mayor a los 4 millones de dólares en el Estado, son en su totalidad hombres. En alusión a esto, Briseño, Briseño y López (2016) refieren que, en los países en vías de desarrollo, las mujeres son educadas en un ambiente socio-cultural conservador adverso, que trae consigo una falta de seguridad en ellas y de la sociedad en la cual han vivido. Esta realidad se traduce en resistencia por parte de la familia para financiar la empresa de una mujer, la renuencia de la banca para asumir riesgos en proyectos creados por mujeres, y la renuencia general para aceptar a las mujeres como responsables para la toma de decisiones o para presentarse como garantías para pedir préstamos para ellas (Briseño et al; 2016).

Se concluye que los hallazgos del estudio son de particular relevancia para los asesores-planificadores financieros, las organizaciones profesionales (de servicios financieros) y los reguladores de la industria. La tolerancia al riesgo debe considerarse durante el proceso de asesoría para que los asesores financieros puedan brindar un trabajo adecuado a sus clientes. Y a la hora de buscar financiamiento para un proyecto, considerar el perfil de los inversionistas sonorenses, su tolerancia al riesgo.

Los inversionistas de mayor edad están preocupados por mantener el ingreso actual, por el escenario local, nacional y mundial, se manifiestan preocupados, con incertidumbre. Los más optimistas, los menos, son los inversionistas jóvenes, quienes están pensando en proyectos de corto y mediano plazo, más no de largo.

\section{REFERENCIAS}

Bernstein, P. (1996). Against the gods: The remarkable story of risk (pp. 1269-1275). New York: Wiley.

Briseño, Olivia; Briseño, Arturo; López, Alfonso (2017). El emprendimiento femenino: un estudio multi-caso de factores críticos en el noreste de México. Innovaciones de Negocios, 2017, XIII, (25), pp. 23-46.

Cordell, David (2001) RiskPACK: How to evaluate risk tolerance. Journal of financial planning, XIV (6), pp. 3656.

Cortés, Joaquin (2016). El nivel de riesgo y el ciclo de vida del inversionista. Mercados y Negocios (34), pp. 107- 
120.

Davies, G.B. (2017). New Vistas in Risk Profiling. CFA Institute Research Foundation. Charlottesville, Estados Unidos.

Finametrica (2015). What Is Risk Tolerance? Recuperado de: https://www.myrisktolerance.com/risktolerance

Glaeser, Edward; Kerr, William; Ponzeto, Giacomo (2010). Clusters of entrepreneurship. Journal of urban economics, LXVII (1), pp. 150-168.

Grable, J.E. (2000). Financial risk tolerance and additional factors that affect risk taking in everyday money matters. Journal of Business and Psychology, 14(4):625-630.

Grable, John (2017). Financial Risk Tolerance: A Psychometric Review. CFA Institute Research Foundation, Charlottesville, Estados Unidos.

Hallahan, A., Faff, R.W. \& Mckenzie, D. 2004. An empirical investigation of personal financial risk tolerance. Financial Services Review, 12(1):57-78.

ICONTEC. (2009). NTC - ISO 31000. Gestión del Riesgo. Principios y Directrices. Bogotá, Colombia: ICONTEC.

Koh, B. \& Fong, W.M. (2011). Personal Fiancial Planning. Jurong: Pearson Education.

Kannadhasan, M; Aramvalarthan, S; \& Goyal, V. (2016). Relationship between biopsychosocial factors and financial risk tolerance: An empirical study. Vikalpa, XLI (2), pp. 117-131.

Klement, Joachim (2018). Risk Profiling and Tolerance: Insights for the Private Wealth Manager, CFA Institute Research Foundation, Charlottesville, Estados Unidos.

Mahmood, Iqbal; Habib Ahmad, Abdul Zahid Khan \& Mansoor Anjum (2011). Behavioral implications of investors for investments in the stock market. European Journal of Social Science, XX (2), pp. 240-247.

Markowitz, Harry. (1953). Portfolio selection. The journal of finance, VII, (1), pp. 77-91.
Meyer, D. y Meyer J. (2007). Measuring Risk Aversión. The journal of risk an insurance, 74(4), pp. 895-906.

Nobre, Lira; Grable, John (2015). "The Role of Risk Profiles and Risk Tolerance in Shaping Client Investment Decisions." Journal of Financial Service Professionals, LXIX, (3), pp. 18-21.

Nguyen, Linh; Gallery, Gerry; Newton, Cameron (2017). The joint influence of financial risk perception and risk tolerance on individual investment decision $\square$ making. Accounting \& Finance, (59), pp. 747-771.

Roszkowski, Marie y and Davey, George ( 2010). Risk perception and risk tolerance changes attributable to the 2008 economic crisis: a subtle but critical difference. Journal of Financial Service Professionals 64, pp. 42-53.

SAGARPA (2015). Datos sector agrícola. México: SAGARPA. Recuperado de https://www.sagarpa. gob.mx/Transparencia/POT 2016/Informe/ CuartolnformeDeLabores SAGARPĀ.pdf

Stangler, Dane (2010) High-growth firms and the future of the American economy. Disponible en SSRN 1568246.

Urteaga, Ernesto; Izagirre, Alfonso (2013). La construcción social del riesgo. EMPIRIA. Revista de metodología de ciencias sociales (25) pp. 147-170.

Weber, Elke; Milliman, Richard (1997). Perceived risk attitudes: Relating risk perception to risky choice. Management science, XLIII(2), pp. 123-144.

Weber, Elke y Klement, Joachim (2018) Risk tolerance and circumstances, en Klement, Joachim (ed.) Risk Profiling and Tolerance: Insights for the Private Wealth Manager, CFA Institute Research Foundation, Charlottesville, Estados Unidos, pp. 55-70. 